\title{
Efficacy and tolerability of carbamazepine for the treatment of painful diabetic neuropathy in adults: a I2-week, open-label, multicenter study
}

This article was published in the following Dove Press journal:

International Journal of General Medicine

2 July 2014

Number of times this article has been viewed

\author{
Tariq Saeed' \\ Muhammad Nasrullah ${ }^{2}$ \\ Adnan Ghafoor ${ }^{3}$ \\ Riaz Shahid ${ }^{4}$ \\ Nadeem Islam ${ }^{5}$ \\ Mohammad Usman \\ Khattak $^{6}$ \\ Neeta Maheshwary ${ }^{7}$ \\ Ahson Siddiqi ${ }^{7}$ \\ Muhammad Athar Khan ${ }^{8}$ \\ 'Pakistan Telecommunication \\ Company Ltd, Karachi, Pakistan; \\ ${ }^{2}$ Cavalary Hospital, Gulberg, Lahore, \\ Pakistan; ${ }^{3}$ Fauji Foundation Hospital, \\ Rawalpindi, Pakistan; ${ }^{4}$ Dr Riaz Shahid \\ Clinic, Peshawar Cantt, Peshawar, \\ Pakistan; ${ }^{5}$ Punjab Employs Social \\ Security Institution, Islamabad, \\ Pakistan; ${ }^{6}$ Medical B Unit, Hayat Abad \\ Medical Complex, Peshawar, Pakistan; \\ ${ }^{7}$ Novartis Pharma Pakistan, Karachi, \\ Pakistan; ${ }^{8}$ Department of Medical \\ Education, King Saud bin Abdulaziz \\ University, Riyadh, Kingdom of \\ Saudi Arabia
}

Correspondence: Neeta Maheshwary

Medical Scientific Liaison,

15 West Wharf Road, Novartis

Pharma Pakistan, Karachi, Pakistan

Tel +92322 8247773

Fax +92 2134376210

Email neeta.maheshwary@novartis.com
Objective: Anticonvulsants are increasingly being used in the symptomatic management of several neuropathic pain disorders. The present observational study was designed to evaluate the efficacy, tolerability, and quality of life (QoL) of carbamazepine use for 12 weeks in patients with painful diabetic neuropathy, in Pakistan.

Methods: This was a 12-week, multicenter, open-label, uncontrolled trial in adult type 2 diabetic patients (aged 18-65 years) suffering from clinically confirmed neuropathic pain (Douleur Neuropathique en 4 [DN4] score $\geq 4$ ). Change in neuropathic pain at week 12 compared with baseline was assessed using the Brief Pain Inventory Scale-Short Form (pain severity score and pain interference score). QoL was determined by the American Chronic Pain Association QoL scale. Safety was assessed based on patient reported adverse events (AEs) and serious AEs.

Results: Of the total 500 screened patients, 452 enrolled and completed the study. The mean ( \pm standard deviation [SD]) pain interference score decreased from $4.5 \pm 2.0$ at baseline to $3.1 \pm 1.9$ at week $12(P<0.001)$. The mean $( \pm \mathrm{SD})$ pain severity score decreased from $5.8 \pm 2.0$ at baseline to $3.6 \pm 2.2$ at week $12(P<0.001)$. There was a decrease of $\geq 30 \%$ in the pain severity score between visits. The mean $( \pm \mathrm{SD})$ QoL scale score improved from $5.9 \pm 1.6$ at baseline to $8.0 \pm 1.7$ at week 12 . A total of ten $(2.2 \%)$ patients reported AEs during the study period. No patient discontinued the study due to AEs.

Conclusion: In this real-life experience study, carbamazepine, when prescribed for 12 weeks to adult diabetic patients suffering from neuropathic pain, showed pain-relief effect, with reduced mean pain severity and mean pain interference scores and with improved QoL and good tolerability profile.

Keywords: anticonvulsant, neuropathic pain, quality of life

\section{Introduction}

Neuropathic pain is a chronic condition caused by damage to or pathological change in the peripheral or central nervous system. The prevalence of chronic pain of neuropathic origin is common in the general population $(6 \%-8 \%)$, as reported in epidemiological surveys. ${ }^{1}$ The International Association for the Study of Pain defines neuropathic pain as "pain arising as a direct consequence of a lesion or disease affecting the somatosensory system". ${ }^{2}$

Painful diabetic neuropathy (PDN) is the most common microvascular complication of diabetes mellitus with peripheral nerve dysfunction. Diabetes affects $8.3 \%$ of the US population, ${ }^{3}$ and $26 \%$ to $47 \%$ of adult patients with diabetes mellitus have been reported to experience neuropathic pain. ${ }^{4}$ Hyperglycemia can be highly correlated to diabetic neuropathy, where the magnitude and duration are proportional to the gradual development and progression of neuropathy. ${ }^{5-7}$ 
Diabetic neuropathy involves small and large sensory fibers and is associated with the symptoms of neuropathic pain that begins in the lower limb, first affecting the toes and then gradually progressing upward. Patients experience numbness, a tingling or burning sensation in the extremities, and further lose the ability to sense pain and temperature. PDN has a major impact on quality of life (QoL) affecting mood, sleep, and daily living activities. ${ }^{7.8}$

In a small population-based study from Wales (UK) of 269 patients who were recruited for assessment, 26.4\% had PDN and a significantly lower QoL (difference in mean scores of 1.7) compared with those with nonneuropathic pain. ${ }^{9}$ In another study, conducted in Turkey, in diabetic patients, $62 \%$ had diabetic neuropathy based on abnormal nerve conduction and clinical examination, and 16\% had neuropathic pain according to the Leeds Assessment of Neuropathic Symptoms and Signs score. ${ }^{10}$ A population-based study in the UK that looked at painful neuropathic characteristics revealed that diabetic nonneuropathic patients of South Asian origin had a 50\% increased risk of developing painful neuropathic symptoms compared with European and African Caribbeans. ${ }^{11}$ Although there is a lack of data from Pakistan, the burden of neuropathic pain in Pakistan is likely to be high, considering the large population and high prevalence of diabetes mellitus and stroke. ${ }^{12}$

Anticonvulsants and antidepressants are among the most commonly used therapeutic options for diabetic neuropathy. A systematic review by Wong et $\mathrm{al}^{7}$ reported that oral tricyclic antidepressants and traditional anticonvulsants were better than the newer generation anticonvulsants for shortterm pain relief. Another work reported that carbamazepine (CBZ), a traditional anticonvulsant, provided pain relief and improved symptoms of patients with PDN. ${ }^{13}$ However, the studies reported were conducted in a small patient population or for a short duration. ${ }^{14-16}$ Thus, the present study was designed to evaluate the efficacy, tolerability, and QoL of CBZ use for a long duration, 12 weeks, in patients with PDN.

\section{Study objectives}

The primary objective of the study was to evaluate the change in neuropathic pain at week 12 compared with baseline, using the Brief Pain Inventory-Short Form (BPI-SF) scale. ${ }^{17}$ The secondary objectives included assessment of the tolerability of CBZ and the QoL of diabetic neuropathic patients after treatment with CBZ. Tolerability was assessed by the incidences of adverse events (AEs) and serious adverse events
(SAEs), and the QoL was assessed using the American Chronic Pain Association QoL scale. ${ }^{18}$

\section{Materials and methods Study design and intervention}

This trial was a 1-year observational, noninterventional, prospective clinical trial with open-label, uncontrolled study design, conducted at multiple sites (Karachi, Lahore, Islamabad, Peshawar, and Rawalpindi) in Pakistan. Type 2 diabetic patients being treated with an oral anti diabetic medication who were aged between 18 and 65 years and who had neuropathic pain (Douleur Neuropathique en 4 [DN4] score $\geq 4)^{19}$ and creatinine clearance $>60 \mathrm{~mL} /$ minute were enrolled in the study (Table 1). The first patient was enrolled on October 7, 2011, and the last patient completed the study on September 29, 2012. The protocol was approved by the ethics committee of Hamdard College of Medicine and Dentistry (HCMD). This study was performed in accordance with the principles of the Declaration of Helsinki and local applicable laws and regulations.

CBZ tablets were administered twice daily orally at the recommended dose of 400-800 $\mathrm{mg}$ daily. The daily dose was titrated gradually from $100 \mathrm{mg}$ (day 1) to $400 \mathrm{mg}$ by the end of the first week (days 6 and 7). The dose was later increased up to $600-800 \mathrm{mg}$ daily, according to the clinical response and the tolerability of the drug. Each patient was followed up for a period of 12 weeks.

\section{Outcome measures}

Change in neuropathic pain at week 12 compared with baseline was assessed by the pain severity score and pain interference scores of the BPI-SF, a self-rated questionnaire. The pain severity score was calculated by taking the mean of scores for pain at its worst, pain at its least, pain on average, and pain experienced at a particular time point. The

Table I Baseline characteristics of patients

\begin{tabular}{ll}
\hline Characteristics & Mean (SD)* \\
\hline Sex, $\mathrm{n}(\%)$ & $288(63.7)$ \\
$\quad$ Male & $164(36.3)$ \\
Female & $48.1 \pm 11.3$ \\
Age, years & $173.6 \pm 68.1$ \\
FPG, $\mathrm{mg} / \mathrm{dL}$ & $10.5 \pm 6.5$ \\
Diabetes, years & $1.65 \pm 0.91$ \\
Creatinine, mg/dL & $40.5 \pm 14.8$ \\
ALT, mg/dL &
\end{tabular}

Notes: $\mathrm{N}=452$. *Data are expressed as mean $( \pm S D)$ unless otherwise specified. Abbreviations: ALT, alanine aminotransferase; FPG, fasting plasma glucose; SD, standard deviation. 
pain interference score was calculated by taking the mean of scores for the interference of pain with general activity, mood, walking, normal work, relations, sleep, and enjoyment of life. The QoL was evaluated, at baseline and week 12, using the American Chronic Pain Association QoL scale. ${ }^{20}$

\section{Statistical analysis}

Data collected were analyzed using SPSS Statistics for Windows, Version 17 (SPSS Inc., Chicago, IL, USA). It was estimated that considering an error rate of $4.5 \%$, a sample size of 474 would be required to yield $95 \%$ confidence. Allowing for some patient dropout, a total of 500 patients were considered sufficient to produce statistical significance. Descriptive analysis of all baseline variables was performed. The results were presented as mean \pm standard deviation (SD) and range, for continuous variables, and as frequency with $95 \%$ confidence interval, for categorical variables. Student's $t$-test for paired samples was used to compare changes in the means of BPI-SF scores at baseline and week 12 .

\section{Results}

Out of the 500 patients screened, 452 (90.4\%) patients were enrolled and completed the study. The majority of the patients were male (63.7\%), with a mean age of 48.1 years (Table 1 ). At baseline, $15.9 \%$ of patients experienced retinopathy, and $11.6 \%$ were suffering from kidney disease. The mean duration of diabetes was 10.5 years and neuropathic mean $( \pm \mathrm{SD})$ pain severity score at baseline was $5.8 \pm 2.0$.

After 12 weeks of CBZ treatment, about 330 (73\%) of the patients reported at least $50 \%$ relief in pain, whereas three $(0.7 \%)$ patients did not report any pain relief. For $32(7.0 \%)$ patients, $100 \%$ pain relief was observed. Moreover, at week 12 , there was a significant $(P<0.001)$ decrease in the mean $( \pm \mathrm{SD})$ pain severity score, from $5.8 \pm 2.0$ at baseline to $3.6 \pm 2.2$ at week 12 , and mean $( \pm \mathrm{SD})$ pain interference score, from $4.5 \pm 2.0$ at baseline to $3.1 \pm 1.9$ at week 12 (Figure 1). A decrease of $>30 \%$ in the pain severity score and pain interference score was observed between the visits. The mean $( \pm$ SD) QoL scale score was significantly $(P<0.001)$ improved, from $5.9 \pm 1.6$ at baseline to $8.0 \pm 1.7$, at week 12 (Figure 1).

Ten (2.2\%) patients experienced at least one AE during the study period (Table 2). The most common AEs were drowsiness, headache, mild dizziness, and nausea. Except for drowsiness, which was considered as drug-related, none of the other AEs were considered to be related to the study. Most of the AEs were mild in severity. No deaths or other SAEs were reported.

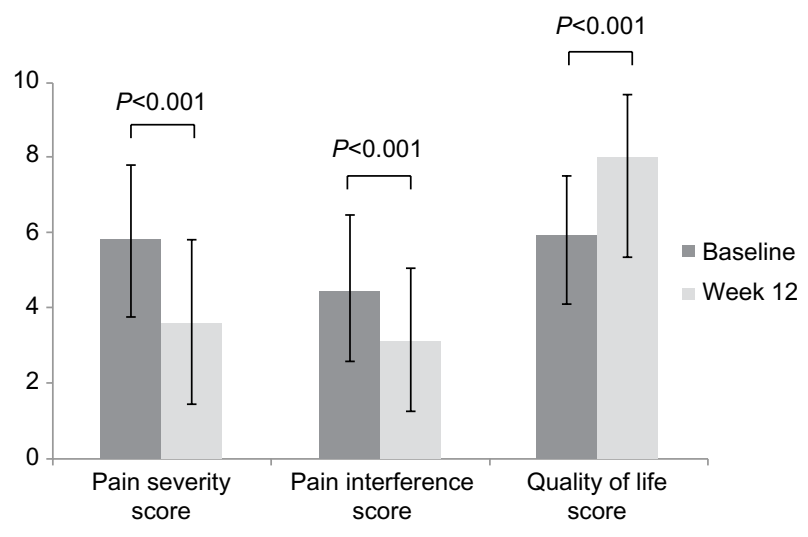

Figure I Change in neuropathic pain and quality of life (QoL) from baseline to week 12.

Note: The data represent pain severity, pain interference, and QoL scores at baseline and week 12 .

\section{Discussion}

This study assessed the efficacy, tolerability, and impact on QoL of treatment with CBZ, in adult patients with diabetic neuropathy. Results of the present study showed that CBZ provided at least $50 \%$ pain relief in more than $70 \%$ of the patients by the end of 12 weeks of treatment. A significant reduction in mean pain severity and mean pain interference scores was observed from baseline to week 12 . The decrease in pain severity and pain interference scores was $\geq 30 \%$ between the visits. Previously, traditional anticonvulsants, such as phenytoin $(100 \mathrm{mg})$, have been studied in the treatment of peripheral neuropathies in small populations and demonstrated improved pain relief compared with placebo..$^{21,22}$ In one double-blind, placebo-controlled trial in 40 patients over a period of 4 weeks, CBZ significantly improved pain from baseline $(P<0.01)$ compared with placebo (both patient and observer assessment). ${ }^{15}$ In another active controlled trial, CBZ improved paresthesia and pain in diabetic neuropathic patients compared with the combination of nortriptyline and fluphenazine, although the difference between the treatment groups was not statistically significant. ${ }^{21}$ The findings of our study are consistent with the results of previous studies in which CBZ improved pain relief. ${ }^{15,21}$

QoL is substantially impaired in diabetic patients with PDN versus diabetic patients without PDN. ${ }^{9}$ PDN interferes with basic daily life activities and decreases QoL. ${ }^{23}$ Davies

Table 2 Adverse events (AEs) during the study period

\begin{tabular}{ll}
\hline $\mathbf{A E}$ & $\mathbf{n ~ ( \% )}$ \\
\hline Drowsiness & $4(0.9)$ \\
Headache & $3(0.7)$ \\
Mild dizziness & $1(0.2)$ \\
Nausea & $2(0.4)$ \\
\hline
\end{tabular}


et al revealed that clinical manifestations of diabetic neuropathy have a significant negative impact on QoL. ${ }^{9}$ Previous studies using CBZ and gabapentin reported that improvement in pain results in substantial improvement in sleep quality and increased mobility, general activity, and mood. ${ }^{15,24}$ In our study, CBZ significantly improved the QoL score in diabetic patients with PDN. Moreover, the patients were able to work/ volunteer for at least 6 hours daily and also had energy to make plans for one evening of social activity during the week and were active over the weekends. The safety profile of CBZ in this study was consistent with earlier studies. ${ }^{14,25}$ Only ten patients $(2.2 \%)$ experienced one or more AE during the study period. The most frequent AEs reported during the study period were drowsiness, headache, mild dizziness, and nausea.

A recent systematic review has suggested that the use of traditional anticonvulsants, such as CBZ, should be preferred over the newer generation anticonvulsants. ${ }^{3}$ Cepeda and Farrar further revealed that traditional anticonvulsants and antidepressants are reported to be cost effective compared with the newer anticonvulsants, such as gabapentin, in the treatment of neuropathic pain of diabetic or postherpetic origin. ${ }^{26}$

To our knowledge, this is the first real-life experience study of 12 weeks duration to prospectively evaluate the efficacy, tolerability, and QoL of CBZ use in a large population with PDN, in Pakistan. This study demonstrated that CBZ was effective in reducing pain and improving QoL in patients with PDN. A limitation of this study was the openlabel and noninterventional study design. A strong placebo effect was previously observed in a randomized, placebocontrolled study of oxcarbazepine in PDN; ${ }^{27}$ however, the contribution of placebo effect in the efficacy of CBZ could not be denied in the present study. Nevertheless, given the prospective real-life setting and the high number of patients enrolled, this data provides compelling evidence for the use of CBZ in patients with PDN. To establish the efficacy of CBZ in PDN, further large scale, double-blind, randomized, placebo-controlled studies are required.

\section{Conclusion}

The results of this real-life experience study in adult patients with PDN, in Pakistan, showed that CBZ provided pain relief, with significant reduction in mean pain scores and with improved QoL and a good tolerability profile.

\section{Acknowledgments}

Authors thank Srujana Takkallapally and Venugopal Peta, professional medical writers (Novartis Healthcare
Private Limited), for writing and editorial assistance. The study was funded by Novartis Pharma Pakistan, Karachi, Pakistan.

\section{Author contributions}

Tariq Saeed participated in the study design and in data collection and critical review. Muhammad Nasrullah and Nadeem Islam performed data collection and analysis and wrote the description of the results. Riaz Shahid, Adnan Ghafoor, and Mohammad Usman Khattak participated in data collection and wrote the Discussion. Ahson Siddiqi participated in design of the study and critical review. Neeta Maheshwary performed data management and data review. Muhammad Athar Khan performed data analysis. All authors participated in the drafting of the manuscript. All authors read and approved the final manuscript.

\section{Disclosure}

The sponsor of the study, Novartis Pharma Pakistan, was involved in study design, data management, data review, drafting and critical review of the manuscript.

NM and AS are full-time employees of Novartis Pharma Pakistan. The authors report no other conflicts of interest.

\section{References}

1. Freynhagen R, Bennett MI. Diagnosis and management of neuropathic pain. BMJ. 2009;339:b3002.

2. Treede RD, Jensen TS, Campbell JN, et al. Neuropathic pain: redefinition and a grading system for clinical and research purposes. Neurology. 2008;70(18):1630-1635.

3. National Diabetes Information Clearinghouse (NDIC). National diabetes statistics. Fast facts on diabetes. National Diabetes Information Clearinghouse; 2011 [updated September 9, 2011; cited Feb 2011]. Available from: http://diabetes.niddk.nih.gov/dm/pubs/ statistics/\#Nervous. Accessed October 30, 2013.

4. Barrett AM, Lucero MA, Le T, Robinson RL, Dworkin RH, Chappell AS. Epidemiology, public health burden, and treatment of diabetic peripheral neuropathic pain: a review. Pain Med. 2007; 8 Suppl 2:S50-S62.

5. Fowler MJ. Microvascular and macrovascular complications of diabetes. Clinical Diabetes. 2008;26(2):77-82.

6. Huizinga MM, Peltier A. Painful diabetic neuropathy: a managementcentered review. Clinical Diabetes. 2007;25(1):6-15.

7. Wong MC, Chung JW, Wong TK. Effects of treatments for symptoms of painful diabetic neuropathy: systematic review. BMJ. 2007; 335(7610):87.

8. Lindsay TJ, Rodgers BC, Savath V, Hettinger K. Treating diabetic peripheral neuropathic pain. Am Fam Physician. 2010;82(2):151-158.

9. Davies M, Brophy S, Williams R, Taylor A. The prevalence, severity, and impact of painful diabetic peripheral neuropathy in type 2 diabetes. Diabetes Care. 2006;29(7):1518-1522.

10. Erbas T, Ertas M, Yucel A, Keskinaslan A, Senocak M; TURNEP Study Group. Prevalence of peripheral neuropathy and painful peripheral neuropathy in Turkish diabetic patients. J Clin Neurophysiol. 2011; 28(1):51-55.

11. Abbott CA, Malik RA, van Ross ER, Kulkarni J, Boulton AJ. Prevalence and characteristics of painful diabetic neuropathy in a large community-based diabetic population in the UK. Diabetes Care. 2011;34(10):2220-2224. 
12. Khealani BA, Nauman A, Khoso NA, et al. Guidelines for Evaluation and Management of Neuropathic Pain. Karachi: Pakistan Society of Neurology; 2010. Available from: http:/www.pakneurology.net/images/ Neuropathic_pain_guidelines_detailed_version.pdf. Accessed October 30, 2013.

13. Wiffen PJ, Derry S, Moore RA, Kalso EA. Carbamazepine for chronic neuropathic pain and fibromyalgia in adults. Cochrane Database Syst Rev. 2014;4:CD005451.

14. Rull JA, Quibrera R, González-Millán H, Lozano Castañeda O. Symptomatic treatment of peripheral diabetic neuropathy with carbamazepine (Tegretol): double blind crossover trial. Diabetologia. 1969;5(4):215-218.

15. Wilton TD. Tegretol in the treatment of diabetic neuropathy. SAfr MedJ. 1974;48(20):869-872.

16. Gómez-Pérez FJ, Choza R, Ríos JM, et al. Nortriptyline-fluphenazine vs carbamazepine in the symptomatic treatment of diabetic neuropathy. Arch Med Res. 1996;27(4):525-529.

17. Iowa Geriatric Education Center. Brief Pain Inventory (Short Form). Iowa City: Iowa Geriatric Education Center; 1991. Available from: http:// www.healthcare.uiowa.edu/igec/tools/pain/briefPain.pdf. Accessed June 4, 2104.

18. American Chronic Pain Association. Quality of Life Scale, A measure of function for people with pain. Rocklin: American Chronic Pain Association; 2003. Available from: http://www.theacpa.org/uploads/ documents/Quality_of_Life_Scale.pdf. Accessed June 4, 2014.
19. Bouhassira D, Attal N, Alchaar H, et al. Comparison of pain syndromes associated with nervous or somatic lesions and development of a new neuropathic pain diagnostic questionnaire (DN4). Pain. 2005;114:29-36.

20. American Chronic Pain Association. Quality of Life Scale: A Measure of Function for People with Pain. Rocklin, CA: American Chronic Pain Association; 2003. Available from: http:/www.theacpa.org/uploads/ Life_Scale_3.pdf. Accessed March 19, 2014.

21. Hussain AM, Afshan G. Use of anticonvulsant drugs for neuropathic painful conditions. J Pak Med Assoc. 2008;58(12):690-696.

22. Chadda VS, Mathur MS. Double blind study of the effects of diphenylhydantoin sodium on diabetic neuropathy. J Assoc Physicians India. 1978;26(5):403-406.

23. Benbow SJ, Wallymahmed ME, MacFarlane IA. Diabetic peripheral neuropathy and quality of life. QJM. 1998;91(11):733-737.

24. Galer BS, Gianas A, Jensen MP. Painful diabetic polyneuropathy: epidemiology, pain description, and quality of life. Diabetes Res Clin Pract. 2000;47(2):123-128.

25. Askmark H, Wiholm BE. Epidemiology of adverse reactions to carbamazepine as seen in a spontaneous reporting system. Acta Neurol Scand. 1990;81(2):131-140.

26. Cepeda MS, Farrar JT. Economic evaluation of oral treatments for neuropathic pain. J Pain. 2006;7(2):119-128.

27. Grosskopf J, Mazzola J, Wan Y, Hopwood M. A randomized, placebo-controlled study of oxcarbazepine in painful diabetic neuropathy. Acta Neurol Scand. 2006;114(3):177-180.
International Journal of General Medicine

\section{Publish your work in this journal}

The International Journal of General Medicine is an international, peer-reviewed open-access journal that focuses on general and internal medicine, pathogenesis, epidemiology, diagnosis, monitoring and treatment protocols. The journal is characterized by the rapid reporting of reviews, original research and clinical studies across all disease areas.

\section{Dovepress}

A key focus is the elucidation of disease processes and management protocols resulting in improved outcomes for the patient.The manuscript management system is completely online and includes a very quick and fair peer-review system. Visit http://www.dovepress.com/ testimonials.php to read real quotes from published authors. 